\title{
Neología e identidad lingüística en el español de México
}

\author{
Neology and linguistic identity in Mexican Spanish
}

\author{
Ramón Felipe Zacarías Ponce de León \\ Universidad Nacional Autónoma de México, México
}

\begin{abstract}
Resumen: Desde un punto de vista onomasiológico, el proceso de acuñación de nuevas palabras está determinado por aspectos socioculturales e identitarios de las comunidades lingüísticas (Štekaver 2005; Körtvélyessy y Štekaver 2014). En el desarrollo de una cultura, la creación de léxico secundario está estrechamente relacionada con el devenir histórico y social de los pueblos. No debe sorprender que en las variedades dialectales de la lengua española puedan encontrarse distintas preferencias en la selección de los esquemas de formación de palabras utilizados para nombrar el mundo. En este artículo, analizaremos los procesos lexicogenésicos del español de México y mostraremos que algunos son propios de esta variedad del español y reflejan aspectos actuales de su sociedad y su cultura. Recurriremos al corpus neológico Morfolex (Zacarías, 2016c), que recopila neologismos formados en México en la última década. Se describirán brevemente los procesos de recolección y clasificación de neologismos y los criterios de productividad que permiten reconocer los esquemas de formación de palabras favoritos. Finalmente, se mostrará que entre los esquemas formativos más productivos y que identifican la variante mexicana se encuentran los sufijos azo, iza, erío; el elemento compositivo narco; las palabras compuestas de nombre y adjetivo, y los cruces léxicos.
\end{abstract}

Palabras clave: neología, formación de palabras, productividad, identidad lingüística.

Abstract: From the onomasiological point of view, the process of coining new words is determined by sociocultural and identity aspects of linguistic communities (Štekaver 2005; Körtvélyessy and Štekauer 2014). In the development of a culture, the creation of secondary lexicon is closely related to the historical and social evolution of peoples. It is not surprising that in the varieties of the Spanish language different preferences can be found in the selection of the word-formation process used to name the world. In this paper, we will analyze that processes in Mexico and show that some are typical of this variety of Spanish and reflect current aspects of their society and culture. We will use the neological corpus Morfolex (Zacarías, 2016c), which compiles neologisms formed in Mexico in the last decade. Briefly describe the processes of collection and classification of neologisms and productivity criteria that allow to recognize the favorite word-formation schemes. Finally, it will be shown that among the most productive schemes are suffixes azo, iza, erío; compositional element narco; compound words of name and adjective $(N+A)$, and blends.

Keywords: neology, word-formation, productivity, linguistic identity.

\section{Introducción}

Los hablantes expresan a través del lenguaje todos los aspectos de su cultura, sus creencias, su historia y sus relaciones sociales. El lenguaje nos brinda las herramientas necesarias para nombrar la realidad y puesto que las relaciones sociales de los pueblos son dinámicas, las estructuras lingüísticas evolucionan continuamente. Esto es más evidente en el léxico, ya que es una necesidad de primera mano poner 
nombre a las cosas. En esta investigación partimos de la hipótesis de que el estudio de la neología de una lengua puede brindar información relevante y actualizada sobre la identidad de un grupo humano. Nos proponemos analizar la neología en el español mexicano y encontrar indicios acerca de la sociedad y la cultura de México. De particular interés será investigar los neologismos acerca del campo semántico de la violencia y el narcotráfico. Por ejemplo, las palabras que aparecen prefijadas con el elemento compositivo narco- son muy frecuentes y reflejan una parte difícil de la realidad de este país. Otros rasgos importantes del español mexicano que se pueden analizar son, la elevada tendencia a acuñar nombres festivos con la composición de nombre y adjetivo (caradura), el uso del colectivo iza con alimentos (taquiza), y la profusión de cruces léxicos (pobresor, de profesor y pobre) entre otros.

Para llevar a cabo lo anterior, recurrimos al corpus morfológico Morfolex que es un proyecto radicado en la Facultad de Filosofía y Letras de la Universidad Nacional Autónoma de México. Este corpus tiene la particularidad de estar etiquetado morfológicamente, lo que permite discriminar y clasificar los neologismos según su estructura morfológica. Cuenta actualmente con 14 mil palabras analizadas y clasificadas, todas recopiladas en el español de México. Con este corpus es posible detectar los patrones de formación de palabras más productivos y observar las tendencias relevantes para la acuñación de neologismos. En el artículo se analizará en primer lugar la relación que existe entre la identidad sociocultural de una comunidad y los procesos de renovación de léxico. Posteriormente, se hará una breve descripción del corpus Morfolex, los procesos de recolección, el etiquetado morfológico y los criterios que permiten reconocer los esquemas de formación de palabras favoritos. Finalmente, se describirán los esquemas de formación de palabras que identifican a la variante mexicana.

\section{Identidad lingüística}

La construcción de la identidad de una comunidad se relaciona siempre con el contexto social y la cultura de los grupos humanos. Debido a esto, en los mecanismos que eligen los individuos para comunicarse se encuentran trazos de su identidad enmarcados en el espacio y en el tiempo que les corresponde. Un aspecto importante de la comunicación es el proceso de denominación de las entidades del mundo, lo que le permite a una comunidad compartir nombres y referirse a las cosas del mismo modo. A esto se refiere Cabré cuando afirma que la creación de nuevas palabras en el discurso "...no suele ser gratuita sino motivada por factores de tipo psicológico [...] y por factores de tipo social" (2016: 39). Por consecuencia, el conjunto de nombres, que utiliza una comunidad se vuelve un reflejo de dichos factores. Son las palabras las que nos permiten "...dar cuenta y darnos cuenta de nuestros grupos de pertenencia, de nuestro ser y estar en el mundo" (Ramos 2000: 421). Para Halliday, el lenguaje y el sistema social están estrechamente relacionados, y para estudiar esta relación crea el concepto de semiótica social, que no es otra cosa que una red de significados, o en palabras del autor: "un sistema de significados que constituye la 'realidad' de la cultura" (Halliday 2001: 161). Visto lo anterior, podemos afirmar que el estudio del léxico no solo tiene un interés teórico dentro de la lingüística, sino que también es útil para dar cuenta "...de la existencia de las diferencias culturales que manifiesta la diversidad lingüística" (Ramos 2000: 431). El léxico es, sin lugar a dudas, el principal reflejo de la 
dinámica de un grupo humano. Por lo tanto, estudiar el lenguaje es estudiar la identidad y la cultura; estudiar la estrecha relación que se establece entre las palabras permite entender el desarrollo de diversos patrones culturales y la variación lingüística presente en todos los ámbitos de la sociedad.

\section{Formación de palabras e identidad lingüística}

Un estudio adecuado de la neología debe tomar en cuenta, además del nivel estructural, que comprende aspectos morfofonológicos y semánticos, los aspectos de uso de la lengua, que abarcan las situaciones socioculturales e identitarias de las comunidades lingüísticas donde se atestiguan los neologismos. A este respecto, Schmid (2011) afirma que los neologismos son acuñados porque los hablantes no pueden encontrar la palabra correcta para la idea que quieren expresar, en la forma que quieren hacerlo, dentro de su comunidad de habla. Para este investigador (2011: 102), el uso de neologismos puede señalar la pertenencia a un grupo social, cuya identidad se reconoce por el uso de las palabras acuñadas dentro de dicho grupo, e incluso puede servir como un mecanismo de exclusión de personas ajenas.

Štekaver (2005: 214) analiza la formación de palabras como una respuesta a la motivación que tienen los hablantes para nombrar las cosas y los hechos del mundo, lo que él llama, the act of naming. Por otro lado, según Körtvélyessy y Štekaver (2014: 412), existen factores que pueden causar considerable variación en la operación de nombrar ya que individualmente pueden seguirse diversas estrategias para acuñar una denominación. Esta situación provoca una competencia entre diversos esquemas de formación de palabras. Con respecto a esta variación, los autores señalan como factores importantes, el estrato social, el nivel educativo, la profesión, la edad y el género. Esta forma de proceder en el análisis del léxico es considerada onomasiológica, es decir, se parte del significado para llegar a la forma de las estructuras lingüísticas. El objetivo de la metodología onomasiológica es, según Štekaver, Varela y Körtvélyessy (2012: 237), investigar la manera en que las categorías cognoscitivas se representan a través de diversos esquemas de formación de palabras. Para estos autores no existe preselección estricta del esquema adecuado a través de reglas lingüísticas.

Según Zacarías (2016a: 26), la selección del esquema de formación de palabras adecuado depende, por un lado, de restricciones lingüísticas, como el significado y la forma de la base de derivación y el significado y la forma del derivado; y, por otro lado, de factores contextuales, como la educación, la edad y el género y la distribución geográfica. Para el autor, no debe olvidarse que, en el proceso de asignación de nombre, los hablantes tienen un papel primordial en la selección del esquema adecuado a partir de un conjunto de esquemas rivales. De acuerdo con lo anterior, podemos afirmar que la identidad lingüística de una comunidad se expresa claramente en la creación de nuevo léxico.

\section{El corpus morfológico}

Morfolex es un corpus léxico etiquetado morfológicamente que se ha construido mediante la recopilación de palabras neológicas acuñadas en el español de México. Se creó con la estructura de una base de datos que contiene diversos campos, entre los que podemos mencionar, el neologismo, su contexto de aparición, la referencias y un conjunto de etiquetas morfológicas y de control. El 
etiquetado morfológico es una característica importante de este corpus porque permite hacer agrupaciones de registros que se refieren a un mismo esquema morfológico; aplicar filtros a la base de datos para crear archivos de trabajo; $y$, aspecto primordial, permite también identificar palabras que aparecen una sola vez en el corpus (hápax legómena). El conteo de las palabras hápax es vital ya que a partir de dichos conteos se realizan cálculos de frecuencia y productividad.

Actualmente, el corpus contiene más de 14 mil palabras etiquetadas morfológicamente y recientemente se ha actualizado con respecto a la última edición del diccionario académico. En (Zacarías, 2016c) se discuten ampliamente todos los aspectos relacionados con el corpus y los fundamentos teóricos de la productividad morfológica. Asimismo, se presentan los resultados del análisis de productividad para todos los esquemas de formación de palabras que se han atestiguado en el corpus.

\section{1. Acopio de neologismos}

La identificación de una palabra como neologismo a partir de una sensación de extrañeza o novedad forma parte de la competencia de los hablantes (Cabré y otros 2002: 161). Estos juicios de neologicidad, sin embargo, son subjetivos ya que varían con cada hablante, lo que impide realizar generalizaciones y haría necesario realizar encuestas amplias para determinar la situación neológica de una palabra. Esta subjetividad convierte este tipo de juicios individuales en una metodología imposible de aplicar cuando se intenta formar un corpus de neologismos. Ante tal imposibilidad, el trabajo en neología utiliza el llamado método lexicográfico. Esta metodología recurre principalmente a listas de exclusión para comprobar el carácter neológico de las palabras. Dichas listas de exclusión se componen de obras lexicográficas: "...los neólogos, por lo general, priorizan el parámetro de la lexicografía para determinar si una unidad es neológica o no" (Fuentes y otros 2009: 107). Lo que se hace es comprobar la ausencia de la palabra candidata a neologismo como requisito indispensable para asignarle el estatus neológico. El criterio lexicográfico puede ser inexacto debido a que los diccionarios van muy a la zaga con respecto a la evolución del léxico y también, a los dispares criterios para incluir nuevas entradas. Sin embargo, como afirma Yañez (2014: 64), dentro del criterio lexicográfico se toman en cuenta también los juicios de los hablantes, puesto que en la selección de un candidato a neologismo interviene la percepción de novedad del hablante en cuanto al desconocimiento de la estructura de la unidad léxica en cuestión. Debido a esto, la metodología a partir de listas de exclusión es la más usual en el trabajo neológico.

Para la elaboración del corpus Morfolex se ha utilizado también el criterio lexicográfico para la asignación del estatus neológico. En este caso, se incluyen diccionarios del español de México. La lista de exclusión incluye, en primer lugar, el Diccionario de la Lengua Española (DLE). Se han considerado las dos últimas ediciones. Recientemente, el corpus neológico se actualizó con la última versión. En segundo lugar, se incluye el Diccionario de Americanismos, editado por la Asociación de Academias de la Lengua Española. Los diccionarios que se refieren a la variedad mexicana son dos. Por un lado, el Diccionario de Mexicanismos, de la Academia Mexicana de la Lengua y, por otro lado, el Diccionario del Español de México, editado por El Colegio de México. 


\section{2. Fuentes de neologismos}

Los procesos lexicogenésicos son puestos en marcha por todos los hablantes, dada la necesidad de crear nuevas denominaciones para diversas entidades, en distintos ámbitos y en toda circunstancia. Por lo tanto, la búsqueda, identificación y recolección de neologismos puede llevarse a cabo tanto en lengua oral como en lengua escrita. Dadas las dificultades inherentes que presenta la recolección oral de palabras, los periódicos han sido una fuente primordial en los trabajos de neología: "Ahora bien, los medios de comunicación y, por ende, la prensa escrita, son los principales propagadores de neologismos, pues reflejan los cambios lingüísticos que se producen en la lengua" (Fuentes y otros 2009: 105). La prensa recoge, sin lugar a dudas, los usos cotidianos del lenguaje y replica en muchos casos las palabras que los hablantes acuñan, tal como lo afirma Cabré: "La prensa escrita supone potencialmente un amplio eco de la sociedad" (2011: 481). En el corpus Morfolex también se recurre a la prensa escrita como fuente principal de neologismos.

\section{El ámbito de la morfología léxica en México}

En esta sección mostraremos que el análisis de la morfología léxica y de los procesos lexicogenésicos de una variedad particular de una lengua nos permite reconocer las preferencias de los hablantes y reflejar rasgos importantes de su identidad y su contexto histórico, social y cultural. Para esto, tomamos algunos de los esquemas productivos del español mexicano y los analizamos a la luz de las costumbres e idiosincrasia de los habitantes de este país. Como ya lo mencionamos líneas arriba, los análisis están basados en los neologismos recogidos en Morfolex.

Es importante mencionar el caso especial de las formaciones apreciativas con el sufijo ito. En primer lugar, siempre se ha reconocido el uso tan extendido de diminutivos en el español general. Particularizando el caso de México, el uso del sufijo ito se ha asociado siempre a la amabilidad típica de los hablantes de este país. Es tan común este sufijo que incluso se le han atribuido características flexivas. Debido a lo anterior, y puesto que ha sido estudiado ampliamente, no lo incluimos en el presente análisis.

Este recuento no pretende ser exhaustivo de ningún modo ya que hay muchos esquemas de formación de palabras que no se han estudiado desde el punto de vista de esta variedad del español. Sin embargo, consideramos que este análisis permite atisbar la estrecha relación que existe entre la morfología léxica y la identidad lingüística de una comunidad.

\section{1. Sufijo iza}

Este sufijo es utilizado para designar valores colectivos: chaviza, pedriza, cachiza, etc. (DEL, s.v.); y golpes: paliza, golpiza, tranquiza, etc. (DEL, s.v.). En el español de México, además de estos valores, se usa también para indicar la abundancia de un alimento (Zacarías, 2015): tamaliza, taquiza, elotiza, etc. En el corpus Morfolex se cuenta con 26 neologismos formados con este sufijo. En la mayoría de los casos, la abundancia de tamales, tacos, elotes, tomates, tortillas y otros alimentos, se suele indicar con este sufijo. Además, es importante mencionar que, en ocasiones, por efecto de la metonimia, estas mismas palabras pueden denominar una fiesta, ya sea religiosa o social. De esta manera, invitar a alguien a una taquiza es convidarlo 
a festejar o a conmemorar un acontecimiento y, posteriormente, como parte de dicho festejo, se ofrece una comida en la que el alimento principal son los tacos.

\section{2. Sufijo erío}

Es otro sufijo colectivo común en la variedad mexicana, pero usado principalmente con connotaciones negativas y despectivas (Zacarías, 2016b). Se usa como alternativa a ería (palabrerío, palabrería; griterío, gritería). Resalta el hecho de que no está definido en el diccionario académico. Moreno de Alba (2013: 62), reconoce varias de estas palabras como americanismos, e incluso, en los casos de derivados de bases con terminación en consonante (camionerío, papelerío, viejerío), afirma que pueden considerarse mexicanismos. En Morfolex, existen 29 neologismos formados con este sufijo, entre ellos, los siguientes: chismerío, ruiderío, animalerío, pobrerío, animalerío, chiverío, etc. En la mayoría de los casos, se utilizan para denominar un colectivo de poco valor o que se desprecia.

\section{3. Sufijo azo}

En la base de datos se cuentan 175 palabras formados con el sufijo azo. La Academia distingue dos valores (NGLE, 2009: 398); en primer lugar, apreciativos, como carrazo; en segundo lugar, acción violenta o golpe, como sillazo. En el corpus, estos últimos son los más numerosos, y, gracias a los neologismos recopilados, podemos observar que en el español mexicano el significado de golpe ha extendido su polisemia para indicar escándalo político y moral, significados estos últimos sumamente productivos: La difícil situación política del país lleva a calificar hechos de corrupción mediante la adjunción de este afijo a nombres de personas y compañías: moreirazo (Moreira, gobernador), elbazo (Elba, secretaria de un sindicato de maestros), pemexazo (PEMEX, la compañía estatal de petróleo), gasolinazo (aumento mensual de la gasolina). Recientemente, también se utiliza para personajes ajenos a la política que generan escándalos como el caso de kalimbazo (Kalimba es un cantante que tuvo cierta fama la década pasada).

\section{4. Circunfijo en- -ar}

Después del sufijo ear, que se utiliza en todos los países de habla hispana, una de las formas predilectas del español mexicano para crear verbos es la parasíntesis con en- -ar (Saucedo 2011). En Morfolex es el circunfijo más frecuente, con 45 palabras neológicas. Se adjunta tanto a sustantivos como a adjetivos para formar verbos con sentido causativo y de cambio de estado. Con sustantivo se interpreta preferentemente como cambio de lugar: encajuelar (poner en una cajuela), enmicar (meter dentro de una mica); con adjetivos se interpreta, por lo regular, como cambio de estado: enchular (hacer que algo se vea chulo, bonito), empanzonar (abultar la barriga o panza).

\section{5. Elemento compositivo narco}

El diccionario académico (s.v.) indica, por un lado, que narco es un elemento compositivo que significa 'droga'. Por otro lado, puede considerarse un cruce léxico en el que dos palabras se mezclan aportando cada una su significado (narcoguerra, la guerra contra el narcotráfico); finalmente, puede interpretarse como un acortamiento de narcotraficante. Desde nuestro punto de vista, esta última es la más adecuada para el contexto mexicano. Dada la lamentable exacerbación de la violencia provocado por el tráfico de drogas, este 
acortamiento se ha vuelto muy productivo. En Morfolex, esta altísima productividad (165 neologismos) ha llevado a clasificarla como prefijo (Zacarías, 2016c). Prácticamente, cualquier sustantivo que haga referencia a entidades relacionadas con el narcotráfico, puede tomar este "elemento": narcomanta, narcoalcalde, narcotienda, narcopolítico, narcoguerra, narcocultura, etc. Tiene amplia preferencia por adjuntarse a sustantivos, en menor medida adjetivos y no se ha atestiguado su uso con verbos.

\section{6. Compuestos $N+A$}

Los compuestos sintagmáticos de nombre y adjetivo, $N+A$, se utilizan para nombrar características sobresalientes de alguna entidad, preferentemente con sentido lúdico o despectivo: mano larga, cabeza hueca, cejagüera, panza verde. Los neologismos formados con este esquema son frecuentes; existen 60 en Morfolex. Sin embargo, este tipo de compuestos no se cuenta entre los más productivos, como pueden ser los de verbo y sustantivo, $\mathrm{V}+\mathrm{N}$ (sacacorchos). Estos compuestos $\mathrm{N}+\mathrm{A}$ son sustantivos que denominan una parte sobresaliente de cualquier entidad para después referirse a esta por metonimia. En el ejemplo, cejagüera es el apodo de una persona que tiene las cejas de ese color (güero es un adjetivo utilizado en el español mexicano para referirse a alguien rubio). Es importante mencionar el contraste que se da con otras variedades del español, que utilizan, para funciones similares, los adjetivos compuestos formados mediante la composición de nombre y adjetivo con vocal de enlace, $\mathrm{Ni}+\mathrm{A}$, o bien la unión de dos adjetivos, también con vocal de enlace, Ai+A (Llanos y Villayandre, 2017). Estos últimos tipos de compuestos, en México, son poco productivos.

\section{7. Cruces léxicos}

Los cruces léxicos son una categoría difícil de analizar y en la mayoría de las gramáticas se le considera un proceso arbitrario por el cual los hablantes construyen palabras sin estructura interna. Sin embargo, llama la atención su alta incidencia en el corpus (309 neologismos). Cortés (2011: 37), ha descrito detalladamente este proceso morfológico y encontrado regularidades que permiten considerarlo un esquema formativo definido y diferenciado, lo que explica su uso tan extendido, sobre todo en el habla coloquial. Estas construcciones son utilizadas en México para denominar entidades con un sentido lúdico a partir de dos características que se traslapan en un contexto particular: candigato (de candidato y gato), robolución (de revolución y robo), estudihambre (de estudiante y hambre).

Los neologismos creados a partir de los esquemas de formación de palabras que hemos discutido representan una parte importante del léxico mexicano actual. Como pudimos observar, estos neologismos reflejan, hasta cierto punto, aspectos importantes del ámbito social y cultural de esta sociedad. La fiesta, entendida en su sentido más general, es una actividad importante en una sociedad que se ha identificado siempre como alegre y bullanguera. Es, por lo tanto, natural que diversas actividades agrupadas alrededor de dichas fiestas sean denominadas específicamente, como en los ejemplos analizados con el sufijo iza. Por otro lado, este carácter alegre se refleja también en las denominaciones lúdicas que se atribuyen tanto a personas como objetos, ya sea con palabras compuestas de nombre y adjetivo, o bien con cruces léxicos. Los aspectos negativos de la sociedad también encuentran expresión en este sistema lingüístico, tal es el caso de la 
corrupción que puede expresarse como un "golpe" moral con el sufijo azo y sobre todo con el tráfico de drogas expresado prefijalmente con narco. Aspectos negativos pueden expresarse también con el colectivo erío muy utilizado para referir despectivamente un conjunto de entidades. Por último, podemos comentar que la alta profusión de verbos neológicos causativos creados con el circunfijo en- -ar caracteriza también al habla mexicana, si bien en este caso no parece tan evidente la conexión con algún aspecto identitario particular.

\section{Conclusiones}

El estudio llevado a cabo indica que la renovación de la morfología léxica está determinada, hasta cierto punto, por aspectos identitarios que son compartidos por los hablantes de una lengua. En el caso que hemos analizado en este artículo, algunas particularidades de la identidad de México que se reflejan en la neología son: la amabilidad, la festividad, la alimentación y los aspectos lúdicos de la vida. Asimismo, también encontramos aspectos negativos que inciden en la sociedad, como la corrupción y la criminalidad, que encuentran expresión neológica específica. Podemos afirmar que un análisis profundo de la morfología léxica, con especial atención en los patrones productivos, revela importantes aspectos de la identidad lingüística de una sociedad.

Estudios como el presentado en esta investigación, fundados en una visión onomasiológica de la morfología lingüística, son importantes porque aportan nuevos datos acerca del alcance de los procesos de formación de palabras, de sus restricciones y preferencias de selección por parte de los hablantes. Asimismo, consideramos que la replicación de un estudio de este tipo -o al menos una búsqueda de los procedimientos de formación de palabras en corpus representativos - en distintas comunidades de habla hispana nos permitiría contar con un panorama de la productividad de los patrones morfológicos en distintas variedades del español.

\section{Bibliografía}

CABRÉ, T. (2011): "La neología y los neologismos: Reflexiones teóricas y cuestiones aplicadas". De la lengua por sólo la extrañeza. Estudios de lexicología, norma lingüística, historia y literatura en homenaje a Luis Fernando Lara. M. VÁZQUEZ, K. ZIMMERMANN y F. SEGOVIA (eds.). México: El Colegio de México, pp. 465487.

- (2016): "Principios y parámetros en una teoría de neologismos". La neología en las lenguas románicas. Recursos, estrategias y nuevas orientaciones. J. GARCÍA, G. DE STERCK, D. LINDER, N. MAROTO, M. SÁNCHEZ, y J. TORRES DEL REY (eds.). Frankfurt: Peter Lang, pp. 27-42.

CABRÉ, T., M. BAYÁ et al. (2002): "Evaluación de la vitalidad de una lengua a través de la neología: a propósito de la neología espontánea y de la neología planificada". Lèxic i Neologia. T. CABRÉ, J. FREIXÁ y E. SOLÉ (eds.). Barcelona: Observatori de Neologia. Institut Universitari de Lingüística Aplicada, Universitat Pompeu Fabra, pp. 159-201. 
CORTÉS, O. (2011): "Formación de palabras y paronomasia". Lingüística Mexicana 6/1, pp. 37-63.

FUENTES, M., C. GERDING et al (2009): "Neología léxica: Reflejo de la vitalidad del español de Chile". Revista de Lingüística Teórica y Aplicada, 47/ 1, pp. 103-124.

HALLIDAY, M.A.K. (2001): El lenguaje como semiótica social. La interpretación social del lenguaje y el significado. Buenos Aires: Fondo de Cultura Económica.

KÖRTVÉLYESSY, L. \& P. ŠTEKAUER (2014): "Derivation in Social Context". The Oxford Handbook of Derivational Morphology. R. LIEBER Y P. STEKAUER (eds.). Oxford/New York: Oxford University Press, pp. 407-423.

LLANOS, L. \& M. VILLAYANDRE (2017): “Gordibuenas' y 'peliteñidos': la productividad del esquema adjetivo/sustantivo + adjetivo con vocal de enlace en la formación de neologismos". La renovación léxica en las lenguas románicas: proyectos y perspectivas. M. SÁNCHEZ, N. MAROTO, G. DE STERCK, J. TORRES DEL REY, D. LINDER, y J. GARCÍA (eds.). Murcia: Editum, 433-444.

MORENO DE ALBA, J. (2013): Notas de gramática dialectal. El Atlas Lingüístico de México. México: UNAM.

RAMOS, E. (2000): "Elementos Léxicos y Construcción de Identidad en el español de Venezuela". Fermentum. Revista Venezolana de Sociología y Antropología, 10/29, pp. 421-432.

REAL ACADEMIA ESPAÑOLA Y ASOCIACIÓN DE ACADEMIAS DE LA LENGUA ESPAÑOLA (2009): Nueva gramática de la lengua española. México: ESPASA. (NGLE)

REAL ACADEMIA ESPAÑOLA Y ASOCIACIÓN DE ACADEMIAS DE LA LENGUA ESPAÑOLA (2014): Diccionario de la lengua española. México: ESPASA. (DLA)

SAUCEDO, H. (2011): "Polisemia en la formación de palabras. El caso de la parasíntesis en en- -ar del tipo enlatar". Lingüística Mexicana, 6, 1, pp. 81-98.

SCHMID, H. (2011): English Morphology and Word-Formation. An Introduction. Berlín: Schmidt Verlag.

ŠTEKAUER, P. (2005): "Onomasiological approach to word-formation". Handbook of Word-Formation. P. ŠTEKAUER Y R. LIEBER (eds.). Dordrecht: Springer, pp. 207232.

ŠTEKAUER, P., S. VARELA \& L. KORTVÉLYESSY (2012): Word-Formation in the World's Languages: A Typological Survey. Cambridge: Cambridge University Press.

YAÑEZ, F. (2014): Prensa y neologismos: La naturaleza adaptativa y creativa del léxico. Tesis doctoral. Madrid: Universidad Nacional de Educación a Distancia.

ZACARÍAS, R. (2015): "Análisis morfológico y semántico del sufijo -iza y sus implicaciones lexicográficas". Signo y Seña, 27, pp. 247-266.

- (2016a): "Rivalidad entre esquemas de formación de palabras". Publicaciones del Centro de Lingüística Hispánica Juan M. Lope Blanch, 62. México: UNAM.

- (2016b): "Análisis morfológico, semántico y lexicográfico del sufijo -erío en el español de México". Thesaurus. Revista del Instituto Caro y Cuervo, 58, pp. 3052.

- (2016c): "Morfología léxica en el español actual de México: neología y productividad". Estudios de Lingüística Aplicada, 34/64, pp. 11-31 